\title{
Interior structure models of terrestrial exoplanets and application to CoRoT-7 b
}

\author{
Frank W. Wagner, Frank Sohl, Heike Rauer \\ Hauke Hussmann and Matthias Grott
}

Institute of Planetary Research, German Aerospace Center, Berlin-Adlershof, Germany email: frank.wagner@dlr.de

\begin{abstract}
In this study, we model the internal structure of CoRoT-7 b, considered as a typical extrasolar terrestrial planet, using mass and energy balance constraints. Our results suggest that the deep interior is predominantly composed of dry silicate rock, similar to the Earth's Moon. A central iron core, if present, would be relatively small and less massive $(<15$ wt.\% of the planet's total mass) as compared to the Earth's (core mass fraction 32.6 wt.\%). Furthermore, a partly molten near-surface magma ocean could be maintained, provided surface temperatures were high enough and the rock component mainly composed of Earth-like mineral phase assemblages.
\end{abstract}

\section{Introduction and methodology}

Already more than a dozen low-mass $\left(<15 \mathrm{M}_{\oplus}\right)$ extrasolar planets have been discovered. CoRoT-7 $\mathrm{b}$ is the first exoplanet among those for which the radius and mass have been accurately determined. In units of Earth equivalents, these are $1.68 \pm 0.09 \mathrm{R}_{\oplus}$ (Léger et al. 2009) and $4.8 \pm 0.8 \mathrm{M}_{\oplus}$ (Queloz et al. 2009), respectively. The average compressed density of CoRoT-7 b of $5.6 \pm 1.3 \mathrm{Mg} \mathrm{m}^{-3}$ is thus similar to that of the Earth (5.515 $\mathrm{Mg} \mathrm{m}^{-3}$ ) and suggests a terrestrial-type bulk composition (Queloz et al. 2009). Modeling the interior structure of CoRoT-7 b is an important leap forward in understanding the origin and evolution of terrestrial-type bodies in the solar system and beyond. In the following, we present a set of four-layer interior models that have been used to infer the planet's bulk composition and physical state.

Our one-dimensional model assumes spherically symmetric and fully differentiated planets in hydrostatic and thermal equilibrium. The interior structure of these planets is obtained by solving the mass and energy balance equations in conjunction with an equation of state (EoS) for the internal density distribution (e.g. Sohl \& Schubert 2007). The chosen Vinet EoS facilitates extrapolation to exceptionally high pressures (Hama \& Suito 1996, Valencia et al. 2007). The thermal profile across the lower mantle and core is assumed to be fully adiabatic and thermal boundary layers at pressure-induced phase transitions and chemical boundaries, e.g. the core-mantle boundary, are accounted for by constant temperature jumps according to Earth-like values. A mixing length formulation (Sasaki \& Nakazawa 1986, Abe 1997) is adopted to calculate the radial distribution of temperature within the upper mantle and to simulate a lithospheric structure within the uppermost part. The basic idea behind this concept is that internally generated heat is primarily transfered by vertical motion of fluid parcels which, after migrating for size-dependent characteristic length scales, will entirely loose their individuality.

\section{Results and discussion}

Scaling laws for terrestrial-type exoplanets are obtained and a relationship between radius $R$ and mass $M$ according to $R \propto M^{0.266}$ has been established within one to 
ten Earth masses, in accordance with previous work (e.g. Valencia et al. 2006, Sotin et al. 2007). Model planets are subdivided into an upper mantle composed of olivine and pyroxene in equal amounts, underlain by lower perovskite and post-perovskite mantle layers, and a central $\epsilon$-iron core. The density models of CoRoT-7 b are constrained by the planet's total radius of $1.68 \mathrm{R}_{\oplus}$ (Léger et al. 2009). We consider iron core mass fractions of 5,15 , and 65 wt. $\%$, corresponding to planetary masses of 5.3, 5.8, and $10 \mathrm{M}_{\oplus}$, respectively.

With regards to the recently reported mass range $4-5.6 \mathrm{M}_{\oplus}($ Queloz et al. 2009) for CoRoT-7 b, a Mercury-like (core mass fraction 70 wt.\%) bulk composition can be safely ruled out, and, augmented by significant internal compression, only a small and less massive iron-rich core may be present at depth. It is even conceivable that an iron-rich core is lacking, turning CoRoT-7 b perhaps into a coreless, pure silicate planet (ElkinsTanton \& Seager 2008).

Moreover, we have extended our model approach by including a lithospheric model to better constrain the radial distribution of temperature within the upper mantle. Due to the close proximity of CoRoT-7 b to its primary, a high surface temperature of about $1800 \mathrm{~K}$ can be expected. The comparatively thin lithospheric layer on top of the upper mantle has only a marginal impact on the deeper interior. Comparing the calculated temperature profile with pressure-dependent rock melting temperatures (Gasparik 1994), we find that the uppermost part of the mantle of CoRoT-7 b could be at least partly molten, provided the mantle was composed of mineral phase assemblages of forsterite and enstatite, similar to the Earth's.

\section{Conclusions}

In summary, CoRoT-7 b may represent a dry, rock-rich planet predominantly composed of silicates, similar to the Earth's Moon. An iron-rich core at depth would be small and less massive or even non-existent, suggesting that CoRoT-7 b may have originated in the iron-depleted region beyond the snowline and lost its volatile mass fraction when subsequently moving toward its primary. Furthermore, albeit strongly dependent on environmental conditions and dominant mantle mineralogy, the planet might harbour a near-surface magma ocean with depth extending across the uppermost mantle.

\section{Acknowledgement}

This research is supported by the Helmholtz Alliance "Planetary Evolution and Life".

\section{References}

Abe, Y. 1997, Phys. Earth Planet. Inter. 100, 27

Elkins-Tanton, L. T. \& Seager, S. 2008, ApJ 688, 628

Gasparik, T. 1994, Mineral. Mag. 58A, 321

Hama, J. \& Suito, K. 1996, J. Phys.: Condens. Matter 8, 67

Léger, A., Rouan, D., Schneider, J. et al. 2009, A $\mathcal{E} A$ 506, 287

Queloz, D., Bouchy, F., Moutou, C. et al. 2009, A\& $A$ 506, 303

Sasaki, S. \& Nakazawa, K. 1986, J. Geophys. Res. 91, 9231

Sohl, F. \& Schubert, G. 2007, in: T. Spohn (ed.), Treatise on Geophysics 10 (Amsterdam: Elsevier), p. 27

Sotin, C., Grasset, O., \& Mocquet, A. 2007, Icarus 191, 337

Valencia, D., O'Connell, R. J., \& Sasselov, D. D. 2006, Icarus 181, 545

Valencia, D., Sasselov, D. D., \& O'Connell, R. J. 2007, ApJ 656, 545 